\title{
Quantization of the Nonlinear Schrödinger Equation on the Half Line
}

\author{
Mario Gattobigio \\ Istituto Nazionale di Fisica Nucleare, Sezione di Pisa, Dipartimento di Fisica dell'Università di \\ Pisa Piazza Torricelli 2, 56100 Pisa, Italy \\ Antonio Liguori \\ International School for Advanced Studies, 34014 Trieste, Italy \\ Mihail Mintchev \\ Istituto Nazionale di Fisica Nucleare, Sezione di Pisa, Dipartimento di Fisica dell'Università di \\ Pisa Piazza Torricelli 2, 56100 Pisa, Italy
}

\begin{abstract}
We establish the second quantized solution of the nonlinear Schrödinger equation on the half line with a mixed boundary condition. The solution is based on a new algebraic structure, which we call boundary exchange algebra and which substitutes, in the presence of boundaries, the familiar ZamolodchikovFaddeev algebra.
\end{abstract}

PACS numbers: 03.70.+k, 11.10Kk

SISSA REF 3/98/FM

IFUP-TH $2 / 98$ 
The recent interest in quantum field theory on the half line $\mathbf{R}_{+}=\{x \in \mathbf{R}: x>0\}$ is related to some successful applications in open string theory, dissipative quantum mechanics and impurity problems in condensed matter physics. This paper concerns the quantization of the nonlinear Schrödinger equation (NLS)

$$
\left(i \partial_{t}+\partial_{x}^{2}\right) \Phi(t, x)=2 g|\Phi(t, x)|^{2} \Phi(t, x), \quad g>0,
$$

on $\mathbf{R}_{+}$with the boundary condition

$$
\lim _{x \downarrow 0}\left(\partial_{x}-\eta\right) \Phi(t, x)=0, \quad \eta \geq 0 .
$$

Our main result is the exact second quantized solution of the boundary valued problem $(1,2)$.

When considered on the whole line $(x \in \mathbf{R})$, Eq.(1) gives rise to one of the most popular and extensively studied integrable system, which has been solved [1-4] by the method of inverse scattering transform. Let us summarize briefly those main results in $\mathbf{R}$, which turn out to be relevant for our investigation in $\mathbf{R}_{+}$. A convenient starting point is a classical solution of Eq.(1), discovered time ago by Rosales [5] and reading

$$
\begin{gathered}
\Phi(t, x)=\sum_{n=0}^{\infty}(-g)^{n} \Phi^{(n)}(t, x) \\
\Phi^{(n)}(t, x)=\int \prod_{\substack{i=1 \\
j=0}}^{n} \frac{d p_{i}}{2 \pi} \frac{d q_{j}}{2 \pi} \bar{\lambda}\left(p_{1}\right) \cdots \bar{\lambda}\left(p_{n}\right) \lambda\left(q_{n}\right) \cdots \lambda\left(q_{0}\right) \\
\times \frac{\mathrm{e}^{i \sum_{j=0}^{n}\left(x q_{j}-t q_{j}^{2}\right)-i \sum_{i=1}^{n}\left(x p_{i}-t p_{i}^{2}\right)}}{\prod_{i=1}^{n}\left[\left(p_{i}-q_{i-1}\right)\left(p_{i}-q_{i}\right)\right]}
\end{gathered}
$$

The bar indicates complex conjugation and the integration is defined by the principal value prescription. It is assumed that the function $\lambda$ is such that the integrals in (4) exist and the series (3) converges for sufficiently small $g$. Any Schwartz test function meets for instance these requirements. A remarkable property of the solution $(3,4)$ is that its general structure is preserved by the quantization. Indeed, following [2-4], the quantum solution on $\mathbf{R}$ admits the series expansion (3) and the $n$-th order contribution has a similar form, namely

$$
\begin{gathered}
\Phi^{(n)}(t, x)=\int \prod_{\substack{i=1 \\
j=0}}^{n} \frac{d p_{i}}{2 \pi} \frac{d q_{j}}{2 \pi} a^{*}\left(p_{1}\right) \cdots a^{*}\left(p_{n}\right) a\left(q_{n}\right) \cdots a\left(q_{0}\right) \\
\times \frac{\mathrm{e}^{i \sum_{j=0}^{n}\left(x q_{j}-t q_{j}^{2}\right)-i \sum_{i=1}^{n}\left(x p_{i}-t p_{i}^{2}\right)}}{\prod_{i=1}^{n}\left[\left(p_{i}-q_{i-1}-i \epsilon\right)\left(p_{i}-q_{i}-i \epsilon\right)\right]} .
\end{gathered}
$$

Besides the $i \epsilon$ prescription to contour poles, the new and fundamental feature is the substitution of the the functions $\{\lambda(k), \bar{\lambda}(k)\}$ with the generators $\left\{a(k), a^{*}(k)\right\}$ of the ZamolodchikovFaddeev (ZF) [6] algebra $\mathcal{A}_{R}$ defined by

$$
\begin{aligned}
& a\left(k_{1}\right) a\left(k_{2}\right)-R\left(k_{2}, k_{1}\right) a\left(k_{2}\right) a\left(k_{1}\right)=0, \\
& a^{*}\left(k_{1}\right) a^{*}\left(k_{2}\right)-R\left(k_{2}, k_{1}\right) a^{*}\left(k_{2}\right) a^{*}\left(k_{1}\right)=0, \\
& a\left(k_{1}\right) a^{*}\left(k_{2}\right)-R\left(k_{1}, k_{2}\right) a^{*}\left(k_{2}\right) a\left(k_{1}\right)=2 \pi \delta\left(k_{1}-k_{2}\right),
\end{aligned}
$$


where

$$
R(p, k)=\frac{p-k-i g}{p-k+i g} .
$$

In Eq.(5) $\left\{a(k), a^{*}(k)\right\}$ are taken in the Fock representation (see e.g. [7,8]) $\mathcal{F}_{R}$ of $\mathcal{A}_{R}$, which provides therefore the state space of the quantum NLS. As usual, the $*$-operation is realized as Hermitian conjugation with respect to the scalar product in $\mathcal{F}_{R}$. In order to give a precise meaning of the cubic term in (1), one introduces a normal ordering : ... : between $a, a^{*}$ by putting all annihilation operators $a$ to the right and all creation operators $a^{*}$ to the left, conserving separately the original order among creators and annihilators themselves. The quantum version of Eq.(1) is then obtained by the substitution

$$
|\Phi(t, x)|^{2} \Phi(t, x) \mapsto: \Phi \Phi^{*} \Phi:(t, x),
$$

and can be verified explicitly [4]. An essential point is also that the equal-time canonical commutation relations

$$
\begin{aligned}
& {[\Phi(t, x), \Phi(t, y)]=\left[\Phi^{*}(t, x), \Phi^{*}(t, y)\right]=0,} \\
& {\left[\Phi(t, x), \Phi^{*}(t, y)\right]=\delta(x-y),}
\end{aligned}
$$

hold [4] on a dense domain in $\mathcal{F}_{R}$.

Eq.(5) is the quantum inverse scattering transform for the NLS on $\mathbf{R}$. It allows to reconstruct the off-shell quantum field $\Phi(t, x)$ from the scattering data encoded in the Fock representation $\mathcal{F}_{R}$ of $\mathcal{A}_{R}$. One can show [1-4] in fact, that the relative asymptotic states can be represented by

$$
\begin{aligned}
\left|k_{1}, \ldots, k_{n}\right\rangle^{\text {in }} & =a^{*}\left(k_{1}\right) \ldots a^{*}\left(k_{n}\right) \Omega & & k_{1}>\ldots>k_{n}, \\
\left|p_{1}, \ldots, p_{n}\right\rangle^{\text {out }} & =a^{*}\left(p_{1}\right) \ldots a^{*}\left(p_{n}\right) \Omega & & p_{1}<\ldots<p_{n}
\end{aligned}
$$

where $\Omega \in \mathcal{F}_{R}$ is the vacuum state and $\left\{k_{1}, \ldots, k_{n}\right\}$ and $\left\{p_{1}, \ldots, p_{n}\right\}$ denote the momenta of the incoming and outgoing particles respectively. Varying $n \geq 0$, the linear subspaces generated by the vectors $(11)$ and $(12)$ give rise to the corresponding asymptotic spaces $\mathcal{F}_{R}^{\text {in }}$ and $\mathcal{F}_{R}^{\text {out }}$, which turn out [8] to be separately dense in $\mathcal{F}_{R}$. By means of Eqs.(6) one easily derives the scattering amplitudes, which have the factorized form

$$
\begin{gathered}
{ }^{\text {out }}\left\langle p_{1}, \ldots, p_{m} \mid k_{1}, \ldots, k_{n}\right\rangle^{\text {in }}= \\
\delta_{m n} \prod_{i=1}^{n} 2 \pi \delta\left(p_{i}-k_{n+1-i}\right) \prod_{\substack{i, j=1 \\
i<j}}^{n} R\left(p_{i}, p_{j}\right) .
\end{gathered}
$$

As it can be expected from integrability, these amplitudes vanish unless $n=m$. Moreover, the particle momenta are separately conserved, $p_{i}=k_{n+1-i}, i=1, \ldots, n$. From Eq.(13) we deduce also that the exchange factor $R$ in the ZF algebra $\mathcal{A}_{R}$ is actually the two-body scattering matrix.

At this stage we have enough background for facing the boundary valued problem $(1,2)$ on the half line $\mathbf{R}_{+}$. Its classical integrability has been investigated in [9]. After some algebra one can verify $[9,10]$ that besides Eq.(1), the field $\Phi(t, x)$ defined by Eqs.(3,4) solves also the boundary condition (2), provided that the function $\lambda(k)$ satisfies the reflection condition 


$$
\lambda(k)=B(k) \lambda(-k),
$$

with reflection coefficient given by

$$
B(k)=\frac{k-i \eta}{k+i \eta}
$$

So, as a first tentative to quantize Eqs. $(1,2)$, one may try to keep Eqs.(3,5) and to implement in $\mathcal{A}_{R}$ the analog of condition (14), namely $a(k)=B(k) a(-k)$. It is easily seen however that such a constraint is not compatible with the exchange relations (6) and the two-body bulk scattering matrix (7).

The next natural conjecture at this point is that the quantum solution of Eqs. $(1,2)$ is still of the form $(3,5)$, but for the replacement of $\mathcal{A}_{R}$ with another appropriate algebraic structure $\mathcal{B}_{R}$, called in what follows boundary algebra. It will be shown below that this conjecture is right and the main problem is to determine $\mathcal{B}_{R}$, finding in particular the quantum counterpart of condition (14). Our strategy in analyzing this problem will be as follows. We will first recall the scattering amplitudes corresponding to the two-body bulk scattering matrix (7) and the reflection coefficient (15). From these amplitudes one can recover the underlying boundary algebra $\mathcal{B}_{R}$. The final step will be to check that Eq.(5) in the Fock representation $\mathcal{F}_{R, B}$ of $\mathcal{B}_{R}$ provides the solution of $(1,2,9,10)$.

The scattering theory of one-dimensional integrable systems in the presence of a reflecting boundary has been developed by Cherednik [11] and successfully applied more recently by Ghoshal and Zamolodchikov [12]. The following picture emerges from these investigations. Let $\left|k_{1}, \ldots, k_{n}\right\rangle$ in be an in-state, representing $n$ particles coming from $x=+\infty$ and thus having negative momenta $k_{1}<k_{2}<\ldots<k_{n}<0$. These particles interact among themselves before and after being reflected by the wall at $x=0$, giving rise to an out-state $\left|p_{1}, \ldots, p_{m}\right\rangle$ out composed of particles traveling toward $x=+\infty$ and thus having positive momenta $p_{1}>p_{2}>\ldots>p_{m}>0$. The transition amplitude between these states vanishes unless $n=m$ and $p_{i}=-k_{i}, i=1, \ldots, n$. Therefore, not only the total momentum, but each momentum is separately reflected. According to [11], the scattering amplitude is

$$
\begin{gathered}
{ }^{\text {out }}\left\langle p_{1}, \ldots, p_{m} \mid k_{1}, \ldots, k_{n}\right\rangle^{\text {in }}= \\
\delta_{m n} \prod_{i=1}^{n} 2 \pi \delta\left(p_{i}+k_{i}\right) B\left(p_{i}\right) \prod_{\substack{i, j=1 \\
i<j}}^{n} R\left(p_{i}, p_{j}\right) R\left(p_{i},-p_{j}\right) .
\end{gathered}
$$

The $R$-factors describe the interactions among the particles in the bulk, while the $B$-factors take into account the reflection from the wall. The crucial point now is that there exists an algebra $\mathcal{B}_{R}$ encoding the scattering amplitudes (16), in perfect analogy to the case without boundary, where (13) are related to $\mathcal{A}_{R} \cdot \mathcal{B}_{R}$ is the boundary algebra we are looking for. It plays the main role in the quantum inverse scattering approach to the boundary valued problem $(1,2)$ and has been introduced and investigated in a more general context in [13]. In the specific case under consideration, $\mathcal{B}_{R}$ is generated by $\left\{a(k), a^{*}(k), b(k)\right\}$. These generators satisfy quadratic exchange relations, which can be conveniently grouped in two sets. 
The first one is

$$
\begin{aligned}
a\left(k_{1}\right) a\left(k_{2}\right) & -R\left(k_{2}, k_{1}\right) a\left(k_{2}\right) a\left(k_{1}\right)=0, \\
a^{*}\left(k_{1}\right) a^{*}\left(k_{2}\right) & -R\left(k_{2}, k_{1}\right) a^{*}\left(k_{2}\right) a^{*}\left(k_{1}\right)=0, \\
a\left(k_{1}\right) a^{*}\left(k_{2}\right) & -R\left(k_{1}, k_{2}\right) a^{*}\left(k_{2}\right) a\left(k_{1}\right)=2 \pi \delta\left(k_{1}-k_{2}\right) \\
& +b\left(k_{1}\right) 2 \pi \delta\left(k_{1}+k_{2}\right),
\end{aligned}
$$

and strongly resembles (6), except for the presence of the so called boundary generator $b(k)$ in the right hand side of the last equation. The second set of constraints describes the exchange relations of $b(k)$ and reads

$$
\begin{aligned}
a\left(k_{1}\right) b\left(k_{2}\right) & =R\left(k_{2}, k_{1}\right) R\left(k_{1},-k_{2}\right) b\left(k_{2}\right) a\left(k_{1}\right), \\
b\left(k_{2}\right) a^{*}\left(k_{1}\right) & =R\left(k_{2}, k_{1}\right) R\left(k_{1},-k_{2}\right) a^{*}\left(k_{1}\right) b\left(k_{2}\right), \\
b\left(k_{1}\right) b\left(k_{2}\right) & =b\left(k_{2}\right) b\left(k_{1}\right) .
\end{aligned}
$$

We observe that the coupling constant $g$ enters the algebra directly through the exchange factor $R$, while there is still no reference to the boundary parameter $\eta$ : it determines which of the inequivalent Fock representations of $\mathcal{B}_{R}$ must be chosen. Indeed, as explained in details in [13], a Fock representation $\mathcal{F}_{R, B}$ of $\mathcal{B}_{R}$ is characterized by a vacuum state $\Omega$, such that:

1. $\Omega$ is annihilated by $a(k)$;

2. $\Omega$ is cyclic with respect $a^{*}(k)$;

3. $\Omega$ is an eigenvector of $b(k)$ with eigenvalue $B(k)$.

One must distinguish the c-number reflection coefficient $B(k)$ from the boundary generator $b(k)$, which according to Eqs.(18) does not even commute with $\left\{a(k), a^{*}(k)\right\}$.

Recapitulating, the mere fact that our system has a boundary shows up at the algebraic level, turning the ZF algebra $\mathcal{A}_{R}$ into the boundary algebra $\mathcal{B}_{R}$. According to point 3 above, the details of the boundary condition (2) (namely, the value of the parameter $\eta$ ) enter at the representation level through the reflection coefficient $B(k)$. In the Fock space $\mathcal{F}_{R, B}$ one has [13]

$$
a(k)=b(k) a(-k),
$$

which is the correct quantum analogue of Eq.(14) and descends from a peculiar automorphism of $\mathcal{B}_{R}$.

The connection with Cherednik's scattering theory is obtained through the identification

$$
\begin{aligned}
\left|k_{1}, \ldots, k_{n}\right\rangle^{\text {in }} & =a^{*}\left(k_{1}\right) \ldots a^{*}\left(k_{n}\right) \Omega, & & k_{1}<\ldots<k_{n}<0, \\
\left|p_{1}, \ldots, p_{n}\right\rangle^{\text {out }} & =a^{*}\left(p_{1}\right) \ldots a^{*}\left(p_{n}\right) \Omega, & & p_{1}>\ldots>p_{n}>0 .
\end{aligned}
$$

One finds in fact, that the amplitudes

$$
\left(a^{*}\left(p_{1}\right) \ldots a^{*}\left(p_{m}\right) \Omega, a^{*}\left(k_{1}\right) \ldots a^{*}\left(k_{n}\right) \Omega\right),
$$


where $(\cdot, \cdot)$ is the scalar product in $\mathcal{F}_{R, B}$, precisely reproduce the right hand side of Eq.(16).

The final step of our consideration is to show that inserting in Eq.(5) the generators $\left\{a(k), a^{*}(k)\right\}$ in the Fock representation $\mathcal{F}_{R, B}$ of $\mathcal{B}_{R}$ and restricting to $x>0$, one gets a field $\Phi(t, x)$ which solves Eqs. $(1,2)$ and satisfies the commutation relations $(9,10)$. The details in verifying the validity of this statement are given in [10]. Here we shall focus on the essential points only. First of all, we would like to fix an appropriate domain $\mathcal{D} \subset \mathcal{F}_{R, B}$ for the quantum fields. For this purpose we denote by $\mathcal{D}^{n}$ with $n \geq 1$ the $n$-particle subspace

$$
\left\{\int d p_{1} \ldots d p_{n} f\left(p_{1}, \ldots, p_{n}\right) a^{*}\left(p_{1}\right) \ldots a^{*}\left(p_{n}\right) \Omega: f \in \mathcal{S}\left(\mathbf{R}^{n}\right)\right\} .
$$

Setting $\mathcal{D}^{0}=\{v \Omega: v \in \mathbf{C}\}$ we then define $\mathcal{D}$ as the linear space of sequences $\varphi=\left(\varphi^{(0)}, \varphi^{(1)}, \ldots, \varphi^{(n)}, \ldots\right)$ with $\varphi^{(n)} \in \mathcal{D}^{(n)}$ and $\varphi^{(n)}=0$ for $n$ large enough. The last condition and the normal ordered structure of $\Phi^{(n)}(t, x)$ directly imply that the series (3) converges in mean value on $\mathcal{D}$ for any $g>0$. By cyclicity of the vacuum, $\mathcal{D}$ is dense in $\mathcal{F}_{R, B}$ and one can show [10] that also Eqs. $(1,2)$ are satisfied in mean value on $\mathcal{D}$. In fact,

$$
\begin{gathered}
\left(i \partial_{t}+\partial_{x}^{2}\right)\left(\varphi_{1}, \Phi(t, x) \varphi_{2}\right)=2 g\left(\varphi_{1},: \Phi \Phi^{*} \Phi:(t, x) \varphi_{2}\right), \\
\lim _{x \downarrow 0}\left(\partial_{x}-\eta\right)\left(\varphi_{1}, \Phi(t, x) \varphi_{2}\right)=0
\end{gathered}
$$

hold for any $\varphi_{1}, \varphi_{2} \in \mathcal{D}$ and $x>0$. The proof of Eq.(20) is similar in spirit to that given by Davies [4] for the NLS on $\mathbf{R}$. The novelty consists in evaluating the contributions of the boundary generator $b$ stemming from the exchange of $a$ with $a^{*}$. In this respect the conditions $x>0$ and $\eta \geq 0$ show to be essential.

As it should be expected, the fields $\Phi(t, x)$ and $\Phi^{*}(t, x)$ have to be regarded as sesquilinear forms on $\mathcal{D}$. In order to deal with operators, one has to take the averages

$$
\Phi(t, h)=\int d x \Phi(t, x) h(x), \quad \Phi^{*}(t, h)=\int d x \Phi^{*}(t, x) h(x),
$$

with $h \in \mathcal{S}(\mathbf{R})$ and such that $\operatorname{supp} h \subset \overline{\mathbf{R}}_{+}$. One has by construction

$$
\begin{gathered}
\Phi(t, h) \mathcal{D}^{(0)}=0, \quad \Phi(t, h) \mathcal{D}^{(n)} \rightarrow \mathcal{D}^{(n-1)}, \quad n \geq 1, \\
\Phi^{*}(t, h) \mathcal{D}^{(n)} \rightarrow \mathcal{D}^{(n+1)}, \quad n \geq 0 .
\end{gathered}
$$

$\mathcal{D}$ is a common invariant domain for the operators $\Phi(t, h)$ and $\Phi^{*}(t, h)$, where the equal-time commutation relations

$$
\begin{aligned}
& {\left[\Phi\left(t, h_{1}\right), \Phi\left(t, h_{2}\right)\right]=\left[\Phi^{*}\left(t, h_{1}\right), \Phi^{*}\left(t, h_{2}\right)\right]=0,} \\
& {\left[\Phi\left(t, h_{1}\right), \Phi^{*}\left(t, h_{2}\right)\right]=\int d x h_{1}(x) h_{2}(x),}
\end{aligned}
$$

hold [10]. Moreover, the vacuum $\Omega$ is cyclic also with respect to $\Phi^{*}(t, h)$ for fixed $t$.

Concerning the time-evolution, one can define on $\mathcal{D}$ the essentially self adjoint operator

$$
H=\int \frac{d p}{2 \pi} \frac{p^{2}}{2} a^{*}(p) a(p),
$$


which generates

$$
\mathrm{e}^{i H t} a(k) \mathrm{e}^{-i H t}=\mathrm{e}^{-i k^{2} t} a(k), \quad \mathrm{e}^{i H t} b(k) \mathrm{e}^{-i H t}=b(k)
$$

Now, it is easily seen that

$$
\Phi(t, h)=\mathrm{e}^{i H t} \Phi(0, h) \mathrm{e}^{-i H t}
$$

which shows that $H$ is actually the Hamiltonian of the NLS model on the half line. Notice the simple form (23) of $H$ in terms of $\left\{a(k), a^{*}(k)\right\}$ and the fact that $b(k)$ does not evolve in time.

The nontrivial correlation functions of our system involve equal number of $\Phi$ and $\Phi^{*}$. From the structure of Eq.(5) it follows that for computing the exact $2 n$-point function one does not need all terms in the expansion (3), but at most the $(n-1)$-th order contribution. For instance, the two-point function is given by

$$
\begin{gathered}
\left(\Omega, \Phi\left(t_{1}, x_{1}\right) \Phi^{*}\left(t_{2}, x_{2}\right) \Omega\right)= \\
=\int \frac{d p}{2 \pi} \mathrm{e}^{-i p^{2}\left(t_{1}-t_{2}\right)}\left[\mathrm{e}^{i p\left(x_{1}-x_{2}\right)}+B(p) \mathrm{e}^{i p\left(x_{1}+x_{2}\right)}\right],
\end{gathered}
$$

and coincides with that of the non-relativistic free field on half line. In that context $\eta$ parametrizes a family of self-adjoint extensions of the Laplacian on $\mathbf{R}_{+}$. The nontrivial scattering is consistently described by the $2 n$-point correlation functions for $n \geq 2$. These functions differ from the free ones and their on-shell limit leads [10] to the transition amplitudes (16), which completes the picture and concludes our quantum field theory description of the NLS model on $\mathbf{R}_{+}$.

Summarizing, we have shown above that the quantum inverse scattering transform (5) works for solving the boundary valued problem $(1,2)$, provided that the ZF algebra $\mathcal{A}_{R}$ is replaced by the boundary algebra $\mathcal{B}_{R}$. It will be interesting to extend our result to the case $\eta<0$, where the presence of boundary bound states must be taken into account. 


\section{REFERENCES}

[1] E. Sklyanin and L. D. Faddeev, Sov. Phys. Dokl. 23, 902 (1978); E. Sklyanin, ibid. 24, 107 (1979).

[2] H. B. Thacker and D. Wilkinson, Phys. Rev. D 19, 3660 (1979); D. B. Creamer, H. B. Thacker and D. Wilkinson, ibid. 21, 1523 (1980).

[3] J. Honerkamp, P. Weber and A. Wiesler, Nucl. Phys. B 152, 266 (1979).

[4] B. Davies, Journ. Phys. A: Math. Gen. 142631 (1981); Inverse Problems 4, 47 (1988).

[5] R. R. Rosales, Stud. Appl. Math. 59, 117 (1978).

[6] A. B. Zamolodchikov and A. B. Zamolodchikov, Ann. Phys. 120, 253 (1979); L. D. Faddeev, Sov. Scient. Rev. Sect. C 1, 107 (1980).

[7] A. Liguori and M. Mintchev, Commun. Math. Phys. 169, 635 (1995); Lett. Math. Phys. 33, 283 (1995).

[8] A. Liguori, M. Mintchev and M. Rossi, Journ. Math. Phys. 38, 2888 (1997).

[9] A. S. Fokas, Physica D 35, 167 (1989).

[10] M. Gattobigio, A. Liguori and M. Mintchev, The Nonlinear Schrödinger Equation on the Half Line, in preparation.

[11] I. V. Cherednik, Theor.. Math. Phys. 61, 977 (1984).

[12] S. Ghoshal and A. B. Zamolodchikov, Int. Journ. Mod. Phys. A 9, 3841 (1994).

[13] A. Liguori, M. Mintchev and L. Zhao, Boundary Exchange Algebras and Scattering on the Half Line, hep-th/9607085 and Commun. Math. Phys., in press. 